\title{
Computational Aerodynamic Sensitivity Studies for Generic Delta Wing Planforms
}

\author{
Andreas Schütte and Rebeca Nunes Marini \\ DLR - German Aerospace Center, 38108 Braunschweig, Germany, \\ Andreas.Schuette@dlr.de
}

\begin{abstract}
The current investigations look at the vortical flow and aerodynamic performance of a generic sharp leading edge double delta wing with negative strake. The work is divided into three studies regarding grid refinement, sensitivity of the turbulence model and validation of the numerical approach by use of experimental data. The focus is on the predicting the vortical flow and aerodynamic values correctly with the most recent numerical methods. For this purpose the prediction of the vortical flow onset progression and interaction is essential and will be discussed. The target configuration is a generic fighter type wing plan form with fuselage provided by Airbus Defence and Space and is part of a national German research cooperation as well as of a NATO research task group on vortex vortex interaction effects. The present results contributing to the cooperation as a starting point to seal aerodynamic technology gaps for next generation fighter configurations.
\end{abstract}

Keywords: computational fluid dynamics, vortex flow, delta wing aerodynamics

\section{Introduction}

A combat aircraft, such as a fighter, needs to meet certain performance requirements, namely maneuverability and agility in sub-, trans- and supersonic flight. Identification and prediction of in-flight flow situations, that could compromise the aircraft's performance is essential in the design process. As technology advances, computational fluid dynamics (CFD) is progressively included in this process, allowing a better understanding of in-flight flow behavior. The flow separation, vortex formation and vortex system interaction are some examples of flow physics that have knowledge gaps. Several research programs have been established at DLR in Germany for studies regarding these matters. Within a cooperation of DLR, Airbus Defence \& Space and TU Munich as well as in a NATO Science and Technology Organization (STO)Applied Vehicle Technology Panel(AVT) task groups a research program is established. One of the main purposes is a better understanding of the flow topology, vortex vortex interaction effects and the influences on the aerodynamic design to accomplish a sufficient aerodynamic performance at medium to high angle of attack range for symmetric and asymmetric onflow conditions. Incorrect predictions of certain flow structures could 
compromise the aircraft's performance like agility as well as compromising the safety, especially within the off design flight regime. The current work is a starting point to seal technology gaps in the prediction capabilities for future fighter aircraft configurations. Several experimental and computational investigations have been performed over the past years to predict the vortical flow aerodynamics of fighter type aircraft. Among others the investigations by Luckring [1] at NASA LaRC should be mentioned as well as the eperimental work by Hummel and Staudacher [2]. The experimental and numerical work regarding the F-16XL CAWAPI configuration by Lamar [3], Fritz and Cummings [4] as well as Hitzel [5] is important as well as the investigations regarding the $\mathrm{X}-31$ configuration by Schütte et al. [6]. Similar investigations applying standard turbulence models on a double delta wing with fuselage have been provided e.g. by Lei [7]. An update of current challenges and technology gaps addressed to the present research activities on future fighter aerodynamic designs is given by Hitzel at al. [8].

The objectives of the current investigation are to provide information on how the grid refinement influences the results obtained for the same configuration, test parameters and computational model. Moreover, it is also set to conduct a sensitivity study of turbulence models. In particular the potential of the RSM model should be assessed. These two studies are related to vortex location, pressure distribution and interaction. Finally, a comparison with experimental data is provided for evaluation of a best practice in common with a brief discussion on the flow physics and the aerodynamic performance is given.

\section{Generic FFD Configuration}

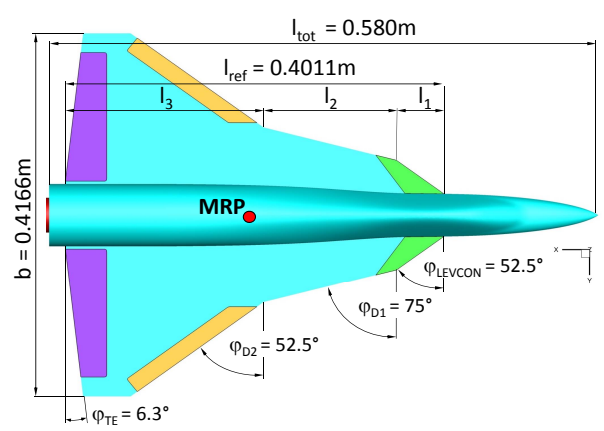

Fig. 1. FFD Reference data.
For the current investigation a generic fighter aircraft configuration has been established to perform the computational simulations. The applied geometry has been given by Airbus Defence Space as a target configuration for the DLR project Diabolo and the NATO STO/AVT research Task Group AVT-316 on Vortex-Vortex Interaction Effects [8]. The geometry is depicted in Figure 1.

The current geometry is a generic double delta wing with negative strake

(Levcon - Leading edge vortex control) and fuselage. To provide a similar setup in computational model like in the wind tunnel tests the rear sting and a cone representing the connection to the wind tunnel support is taken into account for all CFD simulations. The reference length for the pitching moment and Reynolds number is $l_{\text {ref }}$ and for the rolling moment the half span $s$. The exposed wing reference area $A_{\text {ref }}=0.0706 \mathrm{~m}^{2}$ and the moment reference point (MRP) is located at $x=0.4795 \mathrm{~m}, y=0 \mathrm{~m}, z=0.1 \mathrm{~m}$. 


\section{Computational Grids}

Three different grids were applied using the grid generation software CENTAUR by CentaurSoft [9]. Figure 2 shows the overall grid topology of the medium size grid where the resolution of the field sources on the upper side of the wing can be seen quite well. The grid generation strategy is based on a best compromise between coarse and fine grid, in order to accomplish a sufficient grid refinement without losing much accuracy. The major grid parameters are a sufficient first wall spacing

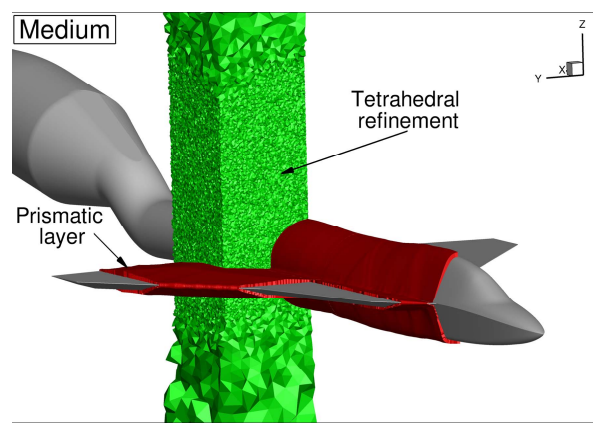

Fig. 2. Medium refinement grid. to achieve overall adequate $y^{+}$values close to the wall, a reasonable resolution of the leading edge to represent the sharp geometry characteristics, a sufficient height of the boundary layer resolving prismatic layer and a sufficient resolution of the tetrahedral field grid over the entire configuration to resolve the vortical flow topology.

The grid size varies from 11.6 to $44.9 \cdot 10^{6}$ grid points for a half model configuration due to changes of the field resolution on the upper wing side. For all grids 30 prismatic layers are applied with a first wall spacing of $0.002 \mathrm{~mm}$ which provides for all grids a sufficient $y^{+}$ distribution of values less than one over the entire upper wing surface. In Figure 3 the velocity profile and the mesh close to the wall is illustrated at a location $y=$ const. The plot shows that the entire boundary layer velocity profile is captured by the prismatic layer.

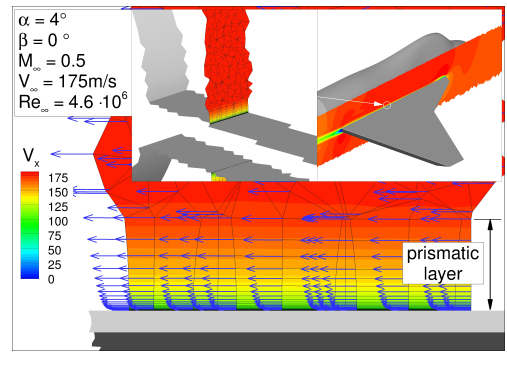

Fig. 3. Velocity vector in streamwise direction close to the wall at a slice $y=$ const. the for medium grid.

\section{Computational and Experimental Approach}

For the present work the RANS flow solver DLR TAU is used. TAU is a CFD software developed by the DLR Institute of Aerodynamics and Flow Technology [10]. The flow solver TAU solves the compressible, three-dimensional, time-accurate Reynolds-Averaged Navier-Stokes equations using a finite volume formulation. The code is based on a hybrid unstructured-grid approach to be able to handle structured and hybrid computational grids, which makes use of the advantages offered by prismatic grid structures applied to resolve the viscous shear layer close to the wall. The current simulations have been performed using the steady state 
and unsteady dual time-stepping approach. The dual time stepping approach was used in case no steady state solution was found. Therefore, the solution is provided by averaging over a certain time period for an unsteady calculation. For the numerical simulations four different turbulence models approaches have been applied as follows. The first one is the one-equation Spalart-Allmaras turbulence model (SA) [11] in its negative formulation. The second turbulence model is the two-equation model k- $\omega$ established by Wilcox [12]. The third model is the TNT model a derivative of the k- $\omega$ model developed by [13] at NLR and the forth model is the RSM (Reynolds-Stress-Model) [14] which closes the Reynolds-averaged Navier-Stokes equations by solving each transport equation from the Reynolds stress tensor plus an equation to solve the turbulence dissipation.

All computational simulations have been performed on the DLR $C^{2} A^{2} S^{2} E$ high performance computing cluster at DLR in Braunschweig. For each simulation 10 nodes with 24 processors each have been used. The computational simulations have been conducted at a Mach number of $M_{\infty}=0.5$ and Reynolds numbers of $R e_{\infty}=3.5$ and $4.6 \cdot 10^{6}$ for an AoA range from $\alpha=2^{\circ}$ to $24^{\circ}$.

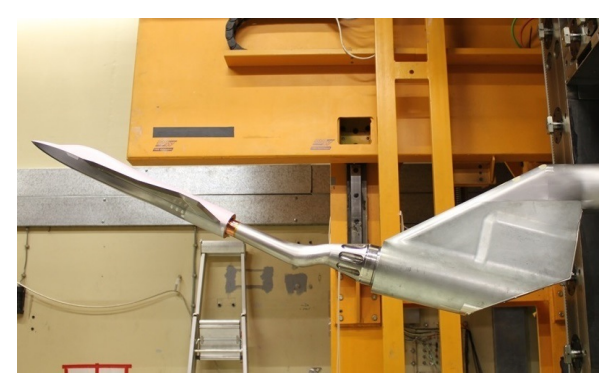

Fig. 4. DLR $\backslash$ Airbus Defence \& Space FFD Wind Tunnel Model in the TWG.

The wind tunnel data for the current investigations have been conducted in German-Dutch Wind Tunnel, Transonic Wind Tunnel in Göttingen (DNWTWG)within a in common wind tunnel campaign of Airbus and DLR. The tests are performed with the DLR FFD model designed by Airbus Defence and Space. The test data used for the current work have been evaluated at a $M_{\infty}=0.5$ and a Reynolds number $R e_{\infty}=3.5 \cdot 10^{6}$. The tests have been performed for an AoA range of $\alpha=10^{\circ}$ to $40^{\circ}$ at symmetric and asymmetric on flow conditions. With this setup forces and moments measurements have been conducted as well as pressure measurements on the upper side of the model by use of PSP - Pressure-Sensitive Paint. A detailed decription of the tests are provided bei Henne et al. [15].

\section{Computational Results}

The computational results are divided into three parts. The first one is the grid sensitivity study discussing the influence of the grid refinement with respect to flow topology, pressure distribution on the upper side of the wing and aerodynamic characteristics. The second study is looking at the influence of the turbulence model applied and the third discusses how well the flow physics and aerodynamic performance is predicted in comparison to experimental data. 


\section{Grid Sensitivity}

The grid sensitivity simulations have been performed using a half model grid at symmetric onflow conditions at a Mach number of $M_{\infty}=0.5$, a Reynolds number of $R e_{\infty}=4.6 \cdot 10^{6}$ and an angle of attack range of $\alpha=2^{\circ}$ to $10^{\circ}$. Figure 5 shows the surface pressure distribution $\left(c_{P}\right)$ and the skin friction lines for the medium grid at an AoA of $\alpha=8^{\circ}$. The flow topology shows three characteristic pressure suction pattern representing at the wing apex the vortex from the negative strake (Levcon vortex), the vortex from the front delta wing (D1 vortex) and finally the vortex from the second delta wing (D2 vortex). The topology for all three grids is plotted in Figure $\mathbf{6}$ shows the pressure distributions at three $x / l_{\text {ref }}=$ const. locations. One is representing the Levcon at $x / l_{\text {ref }}=0.1$, the second at $x / l_{\text {ref }}=0.35$ for the first delta wing and the third at $x / l_{\text {ref }}=0.7$ for the second delta wing. The dashed circles mark the areas of major differences in

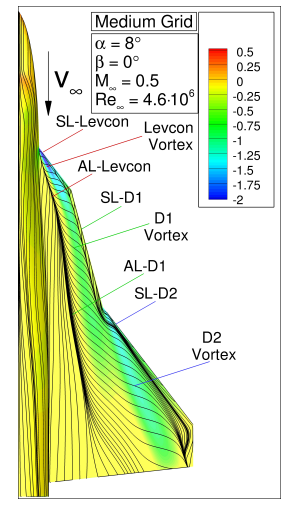

Fig. 5. Surface pressure distribution $c_{P}$ and skin friction lines at an AoA $\alpha=8^{\circ}$ for the medium grid.

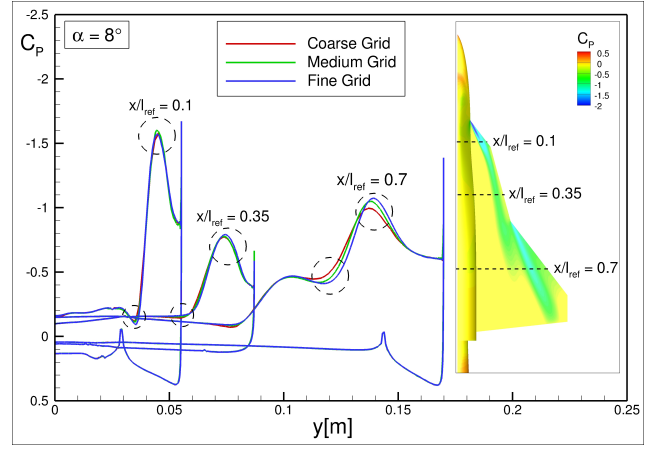

Fig. 6. Comparison of the surface pressure distribution $c_{P}$ at three location $x / l_{\text {ref }}=$ const. at an AoA $\alpha=8^{\circ}$ for the coarse, medium and fine grid.

the $C_{P}$ distribution. Differences can be observed regarding the pressure minimum for each vortex as well as inboard of each suction peak representing the pressure recovery in the area of the attachment line.

Although, there is still a grid dependency and no grid convergence between the medium and fine grid can already considered the differences are reasonable for the current study. With respect to simulation resources the medium grid is chosen for the following studies. However, the validation the grid refinement issue has to be reestablished after a best practice approach is selected.

\section{Turbulence Model Sensitivity}

In the following the influence of the turbulence model will be discussed. The onflow conditions are similar to the previous study. The medium resolution grid is used by applying four different turbulence models: Spalart-Allmaras (SA), k- $\omega$, $\mathrm{k}-\omega$-TNT and the Reynolds-Stress Model (RSM). 


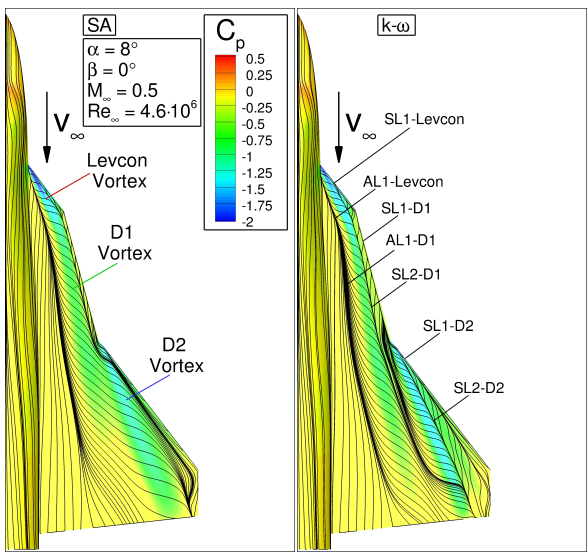

(a)

(b)

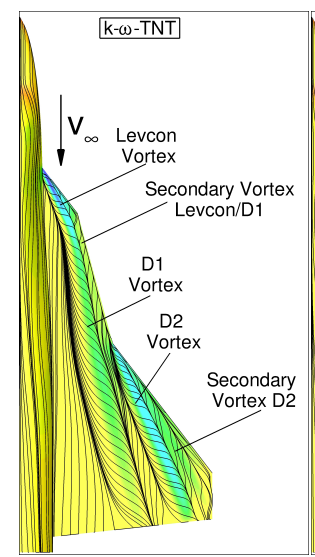

(c)

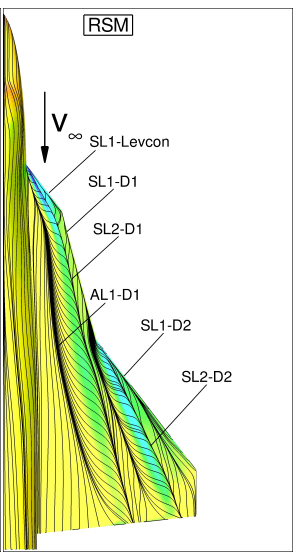

(d)

Fig. 7. $C_{P}$ surface distribution and skin friction lines at an AoA $\alpha=8^{\circ}$ for SA, k- $\omega$, k- $\omega$-TNT and RSM turbulence model.

Figure 7 shows the surface pressure distributions and skin friction lines on the upper side of the model for all four physical models at an AoA of $\alpha=8^{\circ}$. Fig. $7(\mathrm{a})$ is the reference solution conducted with the SA turbulence model similar to Fig. 5. For the solution applying the SA turbulence model in Fig. 7(a) the D1 vortex is providing a distinct low pressure footprint only until $40 \%$ of the length of the second delta wing. Whereas for the other turbulence models a defined low pressure footprint sustains until the trailing edge of the wing, see Fig. 7(b)-(d).

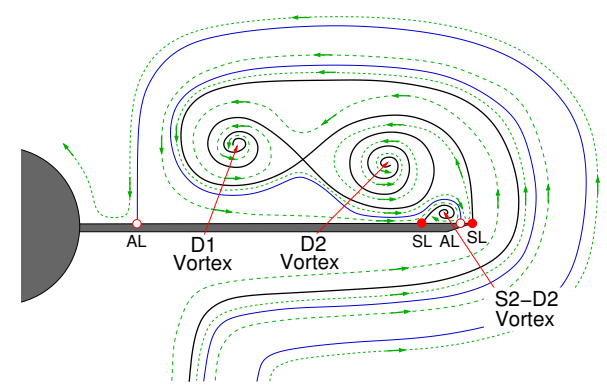

(a)

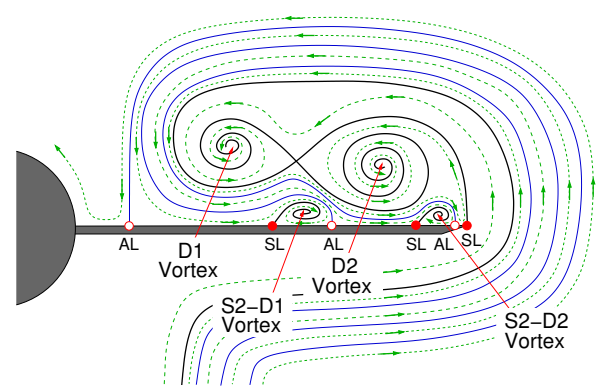

(b)

Fig. 8. Sketch describing the topology of a double branched primary vortex without (a) and with (b) secondary separation of the Delta 1 vortex (b).

In addition the skin friction lines on the upper surface of the second delta wing in Fig. 7(a) are progressing continuously outboard from the attachment line of the first delta wing towards the leading edge. While for the other three models the skin friction lines are discontinuous. This is caused by the secondary vortex of the first delta wing progressing along the second delta wing towards the trailing edge. This flow topology is quite distinct for the solution of the k- $\omega$-TNT 
and RSM model in Fig. 7(c) and (d). Whereas for the k- $\omega$ solution in Fig. 7(b) this structure disappears and the skin friction structure close to the trailing edge is similar to the SA solution.

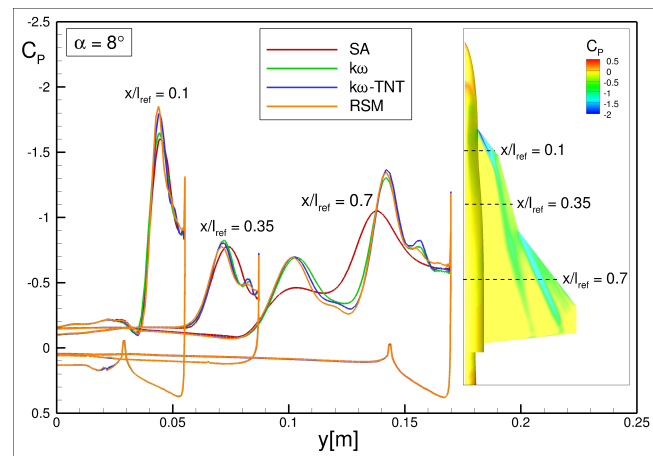

Fig. 9. $c_{p}$ distribution at an AoA $\alpha=8^{\circ}$ for SA, k- $\omega, \mathrm{k}-\omega$-TNT and RSM turbulence model.
Figure 8 shows the difference between the double branched vortex topology without (a) and with remaining Delta 1 vortex secondary separation on the second delta wing (b).

Figure 9 provides the pressure distributions at three $x / l_{\text {ref }}$ constant locations. The plots support the finding observed in the previous figures. The pattern of the $c_{P}$ distribution is similar between $\mathrm{k}-\omega$, $\mathrm{k}-\omega-\mathrm{TNT}$ and RSM but for the SA turbulence model there are significant differences at the $x / l_{\text {ref }}=0.7$ location. At $x / l_{\text {ref }}=0.1$ the location of the pressure minimum for all turbulence models are equal. There is a difference in the peak level which is for SA and k- $\omega$ higher as for k- $\omega$-TNT and RSM. At $x / l_{\text {ref }}=0.35$ a low pressure peak outboard of the primary D1 vortex can be observed which is related to a secondary separation. This peak is not present for SA and not as prominent for RSM and for the two k- $\omega$ approaches. The differences at the location $x / l_{\text {ref }}=0.7$ are most significant. Corresponding to the findings in Fig. 7 there is a particular pressure gradient inboard of the D2 vortex for the k- $\omega$ models and RSM leading to the significant pattern representing an attachment line on the surface. For the SA model this pattern does not exist and the minimum peak of the D2 vortex is much more inboard located as for the other turbulence model approaches.

\section{Validation}

In the following section the computational results will be compared to experimental data to assess the capability of the current approach to predict the complex vortical flow topology and aerodynamic performance correctly and to assess further steps to enhance the considered best practice approach. Figures 10 provides the characteristics of the lift, drag and pitching moment coefficient in comparison to the experiment for symmetric on flow condition for the SA (a), $\mathrm{k} \omega$ (b), k $\omega$-TNT (c) and RSM (d) turbulence model.

The plots show that all turbulence models predict in a reasonable way the lift and drag for AoA $\alpha \leq 14^{\circ}$. The same applies for the pitching moment for the $\mathrm{k} \omega$ models and RSM. Whereas the SA model is providing a to high front loading pitching moment in common with a not matching gradient in comparison to the experiment. For AoA $\alpha>14^{\circ}$ the lift is over-predicted by all models at $\alpha=16^{\circ}$ whereas the characteristics of the RSM model recovers at an AoA of $\alpha=18^{\circ}$. However the values predicted by the SA turbulence model are to high as well as the gradient of the lift coefficient. The same applies for the k $\omega$ and RSM model 
at $\alpha=16^{\circ}$. The steep increase of the pitching moment for AoA $\alpha \geq 16^{\circ}$ is only captured by the RSM approach.

To evaluate the accuracy of the predicted aerodynamic performance a comparison between CFD and experiment regarding the surface pressure distribution is necessary. Figure $\mathbf{1 1}$ shows the comparison between CFD and experiment for the RSM turbulence model at three locations $x / l_{\text {ref }}$. The comparisons to the experiment are given at an AoA of $\alpha=16^{\circ}$ and $20^{\circ}$.

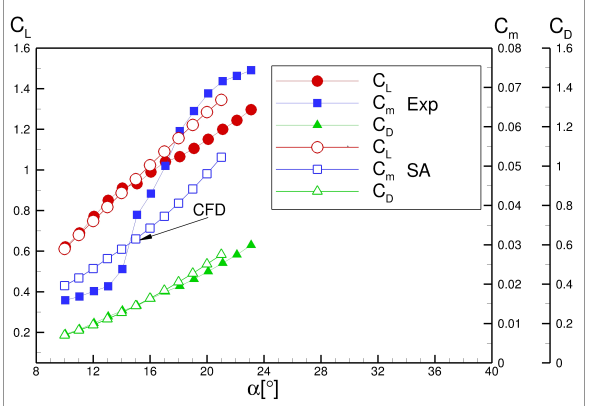

(a)

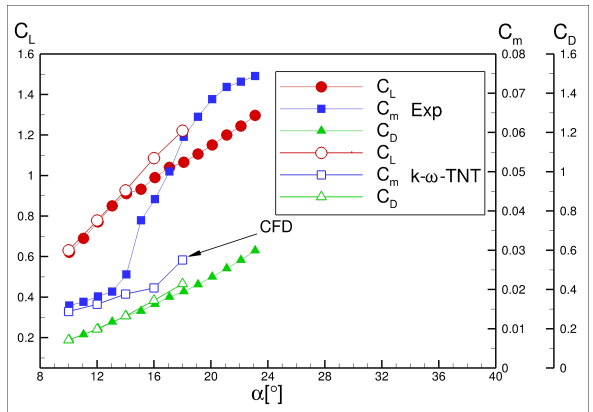

(c)

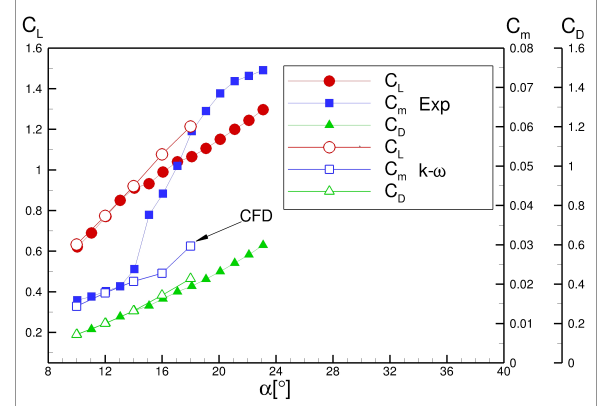

(b)

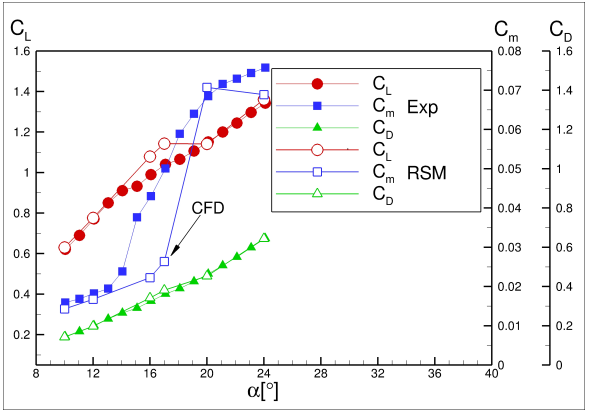

(d)

Fig. 10. Comparison of the calculated lift, drag and pitching moment coefficient versus AoA in comparison to experimental data for all applied turbulence models. $M_{\infty}=0.5$, $R e_{\infty}=3.5 \cdot 10^{6}$ at symmetric onflow condition.

The suction peak of the primary vortex of the first delta wing (D1) is represented well by the RSM model. The location of the vortex of the first delta wing (D1) as well as the secondary separation (S2-D1) is captured well. However, the suction peaks are slightly over-estimated. The same applies for the suction peak of the vortex of the second delta wing D2 at $\alpha=16^{\circ}$. Whereas for $\alpha=20^{\circ}$ the CFD solution matches the suction level inboard of the merged D1 and D2 vortex correctly. With respect to the performance prediction of the present configuration the good agreement between the CFD calculations and experiment applying the RSM model are quite promising especially for higher AoA. Not only the gradient and pitching moment level is capture but also the correct suction level on the upper wing surface and thus the flow topology. Although, additional experimental PIV (Particle Image Velocimetry) data would help to support these 


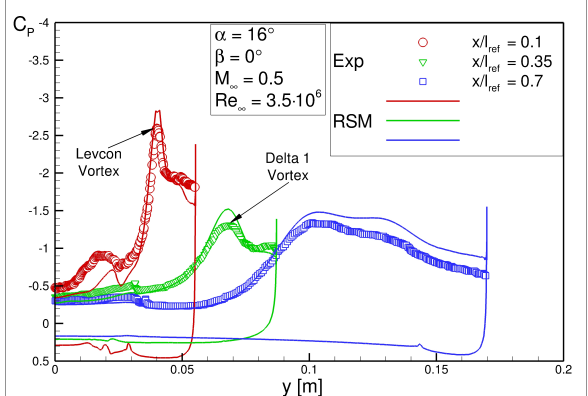

(a)

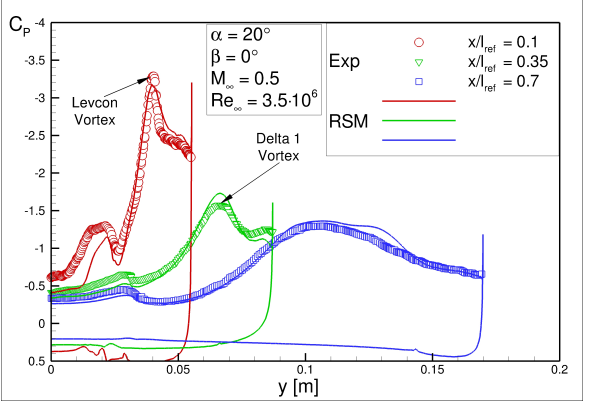

(b)

Fig. 11. $c_{P}$ quantities for an AoA of $\alpha=16^{\circ}$ (a) and $20^{\circ}$ (b) for the RSM turbulence model.

results with respect to the vortex strength and distance towards the surface. All of this is important to get confidence to be able to predict the critical states like vortex breakdown location and $c_{l \beta}$ instabilities which are essential to assess the performance of the stability and control behavior of future fighter aircraft.

\section{Conclusion}

The current investigation provide computational studies to assess the prediction capability of the complex vortical flow and aerodynamic performance of a generic sharp leading edge double-delta-wing fighter type configuration with negative leading edge strakes. The studies included a grid generation sensitivity study, the influence of different turbulence models as well as a validation of the RANS CFD solver DLR TAU by use of experimental data. The investigations have been performed at a Mach number of $M_{\infty}=0.5$ within a Reynolds number range of $R e_{\infty}=3.5 \cdot 10^{6}$ to a $4.6 \cdot 10^{6}$ related to the wind tunnel conditions of the applied configuration.

The grid sensitivity study shows major dependencies on the grid refinement regarding the representation of the suction peaks of the vortices as well as on the pressure recovery in the vicinity of the attachment lines. Although a final grid convergence has not been achieved a medium size grid have been applied. However, a second grid assessment has to be done after selecting a best practice for the turbulence model.

Within the turbulence model sensitivity study it was found that only the pitching moment coefficient was influenced significantly by the grid refinements and selected turbulence models. Major differences regarding the flow topology prediction have been assessed for the Spalart-Allmaras turbulence model in comparison with two versions of the $\mathrm{k}-\omega$ models and the Reynolds-Stress turbulence model.

Finally, the computational results have been compared to experimental data with the scope of a validation and assessment of a best practice approach. The study showed that the SA turbulence is not able to provide the correct flow 
physics and thus the aerodynamic performance. The $\mathrm{k}-\omega$ models provide in some areas reasonable results but deliver in some areas inaccurate solutions for example by over-predicting the suction of the secondary vortices on the upper wing side. The RSM model delivered for the entire AoA and AoS range the best results and can be considered as the turbulence model to chose when proceeding the investigation towards design studies.

Nevertheless, additional grid refinement studies have to be performed as well as extending the angle of attack and side slip as well as the Mach number range to get a comprehensive evaluation of the prediction capabilities of the currently applied RANS solver.

\section{References}

1. Luckring, J.: Aerodynamics of Strake-Wing Interactions. AIAA Journal of Aircraft 16(11), 756-762 (1979)

2. Hummel, D., John, H., Staudacher, W.: Aerodynamic characteristics of wingbodycombinations at high angles of attack. ICAS Proceedings, 14th ICAS Congress Toulouse, FRA 2, 747-762 (1984)

3. Lamar, J.: Prediction of F-16XL Flight-Flow Physics. AIAA Journal of Aircraft 46(2), 354-354 (2009)

4. Fritz, W., Cummings, R.M.: What was learned from the numerical simulations for the VFE-2? AIAA Paper 2008-399 (2008)

5. Hitzel, S.: Sub- and Transonic Vortex Breakdown Flight Condition Simulations of the F-16XL Aircraft. AIAA Journal of Aircraft 54(2), 428-443 (2017)

6. Schütte, A., Boelens, O., Loeser, T., Oehlke, M.: Prediction of the flow around the X-31 aircraft using two different CFD methods. AIAA Paper 2010-4692 (2010)

7. Effect of RANS Turbulence Models on Computation of Vortical Flow over WingBody Configuration. The Japan Society for Aeronautical and Space Sciences 48(161), 152-160 (2005)

8. Hitzel, S., Winkler, A., Hövelmann, A.: Vortex Flow Aerodynamic Challenges in the Design Space for Future Fighter Aircraft. DGLR Fachsymposium STAB, Darmstadt (2018)

9. CentaurSoft: Centaur Tutorial Version 12.0. http://www.centaursoft.com (2017)

10. Schwamborn, D., Gerhold, T., Heinrich, R.: The DLR TAU-Code: Recent applications in research and industry. Proceedings of "European Conference on Computational Fluid Dynamics"ECCOMAS CDF, Delft, The Netherland (2006)

11. Spalart, P., Allmaras, S.: A one-equation turbulence model for aerodynamic-flows. La Recherche Aérospatiale journal, No. 1, pp. 5-21 (1994)

12. Wilcox, D.: Formulation of the $\mathrm{k}-\omega$ turbulence model revisted. AIAA 46.11 pp. 2823-2838 (2008)

13. Kok, J.: Resolving the dependence on free-stream values for the $\mathrm{k}-\omega$ turbulence model. AIAA Journal 38(7), 1292-1295 (2000)

14. Eisfeld, B., Rumsey, C., , Togiti, V.: Verification and Validation of a SecondMoment-Closure Model. AIAA Journal 54(5), 1524-1541 (2016)

15. Henne, U., D. Yorita, D., Klein, C.: Experimental Aerodynamic High Speed Investigations Using Pressure-Sensitive Paint for Generic Delta Wing Planforms. DGLR Fachsymposium STAB, Darmstadt (2018) 